\title{
Hospital readmissions with acute infectious diseases in New Zealand children $<2$ years of age
}

Silvia Seibt ${ }^{1}$, Catherine A. Gilchrist ${ }^{2}$, Peter W. Reed ${ }^{3}$, Emma J. Best ${ }^{2,4}$, Anthony Harnden ${ }^{5}$, Carlos A. Camargo Jr ${ }^{6}$ and Cameron C. Grant ${ }^{2,7^{*}}$ (i)

\begin{abstract}
Background: Infectious diseases are the leading cause of hospital admissions in young children. Hospitalisation with an infectious disease is a recurrent event for some children. Our objective was to describe risk factors for infectious disease readmission following hospital admission with an infectious disease in the first two years of life.

Methods: We performed a national cohort study of New Zealand children, born 2005-2009, with an infectious disease admission before age 24 months. Children readmitted with an infectious disease within 12 months of the first infectious disease admission were identified. Every infectious disease admission was categorised as a respiratory, enteric, skin and soft tissue, urinary or other infection. Independent associations of demographic and child health factors with infectious disease readmission were determined using multiple variable logistic regression.

Results: From 2005 to 2011, there were 69,902 infectious disease admissions for 46,657 children less than two years old. Of these 46,657 children, 10,205 (22\%) had at least one infectious disease readmission within 12 months of their first admission. The first infectious disease admission was respiratory (54\%), enteric (15\%), skin or soft tissue (7\%), urinary (4\%) or other (20\%). Risk of infectious disease readmission was increased if the first infectious disease admission was respiratory $(\mathrm{OR}=1.87,95 \% \mathrm{Cl} 1.78-1.95)$ but not if it was in any other infectious disease category. Risk factors for respiratory infectious disease readmission were male gender, Pacific or Māori ethnicity, greater household deprivation, presence of a complex chronic condition, or a first respiratory infectious disease admission during autumn or of $\geq 3$ days duration. Fewer factors (younger age, male gender, presence of a complex chronic condition) were associated with enteric infection readmission. The presence of a complex chronic condition was the only factor associated with urinary tract infection readmission and none of the factors were associated with skin or soft tissue infection readmission.

Conclusions: In children less than two years old, infectious disease readmission risk is increased if the first infectious disease admission is a respiratory infectious disease but not if it is another infectious disease category. Risk factors for respiratory infectious disease readmission are different from those for other infectious disease readmissions.
\end{abstract}

Keywords: Infant, Child preschool, Cohort studies, Diarrhea, Hospitalization, Patient readmission, Respiratory tract infections, Skin and soft tissue infections, Urinary tract infections

\footnotetext{
* Correspondence: cc.grant@auckland.ac.nz

${ }^{2}$ Department of Paediatrics: Child and Youth Health, Faculty of Medical and

Health Sciences, University of Auckland, Private Bag 92019, Wellesley Street,

Auckland 1142, New Zealand

${ }^{7}$ General Paediatrics, Starship Children's Hospital, Auckland District Health

Board, Auckland, New Zealand

Full list of author information is available at the end of the article
}

(c) The Author(s). 2018 Open Access This article is distributed under the terms of the Creative Commons Attribution 4.0 International License (http://creativecommons.org/licenses/by/4.0/), which permits unrestricted use, distribution, and reproduction in any medium, provided you give appropriate credit to the original author(s) and the source, provide a link to the Creative Commons license, and indicate if changes were made. The Creative Commons Public Domain Dedication waiver (http://creativecommons.org/publicdomain/zero/1.0/) applies to the data made available in this article, unless otherwise stated. 


\section{Background}

Globally, infectious diseases are a leading cause of morbidity and mortality in young children [1]. From 2004 to 2008 in New Zealand (NZ), infectious diseases accounted for $60 \%$ of hospitalisations of children < 5 years old [2]. Most infectious disease related hospitalisations were due to lower respiratory tract, enteric, and skin and soft tissue infections [2].

Hospitalisation rates for infectious diseases are higher in NZ than in other developed countries. Children < 2 years old are more than twice as likely to be hospitalised with bronchiolitis in NZ (2006-2010: 45/1000) than in England (2007-2010: 20/1000), or the United States (US) (2000-2009: 16/1000) [3-5]. Hospitalisation rates for pneumonia in children $<2$ years old in $\mathrm{NZ}$ (2006-2010: 14/1000) are twice those in the US (20072009: 7/1000) [3, 6], and children $<1$ year old have eight times the US rate of skin infection hospitalisation (NZ 2006-2010: 21/1000, US 2005 2.4/1000) [3, 7]. In recent decades the rate of infectious disease hospital admissions in NZ has increased [2]. This represents a true increase in disease incidence, attributed to ethnic and social inequalities, and to disparities in social determinants of health including household income, housing conditions, and access to healthcare [2].

Hospitalisation with an infectious disease (ID) is a recurrent event for some children. In a US study, the $3 \%$ of children with four-or-more recurrent admissions, including but not limited to ID, accounted for $19 \%$ of all paediatric hospital admissions [8]. Identifying strategies to prevent readmission is therefore an important component of quality of care improvement [9].

Early identification of children at risk of ID readmission and prevention of repeated illness episodes may prevent subsequent chronic disease. For example, globally almost two-thirds of children with ID-associated bronchiectasis have a history of recurrent acute lower respiratory infections (ALRIs) [10-12]. Bronchiectasis is particularly prevalent in indigenous children from NZ, Australia, Alaska, and Canada and a history of early infant pneumonia and/or recurrent ALRIs is associated with bronchiectasis in over $90 \%$ of cases $[11,13,14]$.

Our aim was to describe recurrent hospitalisations for infectious diseases in young NZ children, including factors associated with ID readmission. The ability to identify children at risk of recurrent disease when they first present to hospital allows the development and evaluation of interventions targeted to this at-risk group.

\section{Methods}

\section{Study design and setting}

We created a national cohort study of NZ children born over a five-year period (2005-2009) and described their ID hospitalisations. We identified the children hospitalised with an ID during their first 24 months of life, and the subset of children subsequently readmitted with an ID within 12 months of their initial ID hospitalisation were enrolled in the study. The study protocol was reviewed by the NZ Health and Disabilities Ethics Committee, with the ethics committee determining that ethical approval was not required. The NZ Ministry of Health granted data access, providing data with encrypted NHI numbers to maintain patient anonymity. The Ministry of Health did not grant permission for data sharing.

\section{Study population and sample}

Children were eligible if born between 1st January 2005 and 31st December 2009 and a NZ resident at the time of their first ID hospitalisation. Hospitalisation data were collected over six years, from 1st January 2005 to 31st December 2011. Children were excluded if they had an ID admission in their first 24 months of life but died within 12 months of this first admission $(n=117)$. Permission to access data on cause of death was not obtained.

A hospital admission was defined as an overnight stay in a hospital inpatient ward. We excluded episodes where a child was admitted and discharged on the same day. In NZ all acute ID hospital admissions during childhood are to public hospitals.

Hospitalisation data were obtained from the NZ national dataset of hospital admissions, the National Minimum Dataset (NMDS) [15]. We used the national health index (NHI) number, a unique identifier assigned to every person on contact with health services in NZ, to identify all hospitalisations for each child. To maintain anonymity, data provided from the NMDS contained an encrypted unique identifier for each child. Thus, written informed consent was not required.

\section{Identification of infectious disease hospital admissions} Using the approach developed by the US Centers for Disease Control and Prevention [16, 17], and previously used in NZ [2], we defined an ID hospitalisation as one for which the principal International Classification of Disease (ICD-10) code was for an ID (Additional file 1).

Within the NMDS, each hospital admission may include more than one inpatient event. For example, a child admitted to intensive care and then transferred to the inpatient ward is described as two separate events. To ensure each admission was counted only once, consecutive events with the same discharge and admission dates were counted as a single hospitalisation.

\section{Study sample demographics and illness history}

Demographic information was obtained from the NMDS. Ethnicity (European, Māori, Pacific, Asian and 
Other) was defined as that stated by parents at the first admission. Socioeconomic status was defined using a small area unit level descriptor of household deprivation created from national census data (NZDep2006) grouped as quintiles [18]. As defined by Berry et al. (2011), children with complex chronic conditions (CCC) known to be associated with recurrent hospital admission were identified from the ICD-10 codes listed for the first ID admission [8].

\section{Statistical analyses}

ID hospitalisations were grouped into five diagnostic categories: acute respiratory infection (ARI), enteric infection, skin or soft tissue infection (SSTI), urinary tract infection (UTI) and other ID (Additional file 1). Other ID included septicaemia, meningococcal disease, meningitis, osteomyelitis, and septic arthritis. An ID readmission was defined as a second or subsequent hospital admission with an ID principal discharge code, > 1 day after the discharge from the first admission and < 12 months from the original ID admission.

Data were analysed using JMP V10.0 (SAS Inc.). We used multivariable logistic regression to identify independent associations between demographic variables and the risk of hospital readmission with an ID. We determined whether the risk of readmission with an ID varied by type of ID causing the first admission (ARI, enteric, SSTI, UTI or other ID), and, within the ARI group, by type of acute lower respiratory infection (pneumonia, bronchiolitis, influenza and other ARI) (Additional file 2). Within the ARI, enteric, SSTI, UTI or other ID diagnostic groups, we then described the risk factors associated with readmission with a second ID episode within the same ID diagnostic group. Associations were reported using adjusted odds ratios (OR) and 95\% confidence intervals (CI).

\section{Results}

\section{Study population and sample}

There were 659,495 hospital discharge events (from 1st January 2005 and 31st December 2011) for children born between 1st January 2005 and 31st December 2009 (Fig. 1). These events, which included formal transfers and other patient movements, converted to 587,336 hospital admissions, of which 177,545 (30\%) included an ID discharge code. We excluded 34,691 admissions (nonacute, to a private hospital or not a NZ resident); and 40,932 admissions lasting $<24 \mathrm{~h}$. Of the 101,922 acute overnight public hospital ID admissions, for 20,186 the ID discharge code was not the principal code, and for 11,834 the first ID admission occurred at age $\geq 2$ years.

There were 69,902 ID hospital admissions for 46,657 children $<2$ years old, of whom 10,205 (22\%) had at least one subsequent ID readmission within the 12 months following their first admission. Therefore, $15 \%$ of the national birth cohort from 2005 to 2009 had an ID hospital admission before age two years, with $3 \%$ having at least one further ID admission within the next 12 months.

\section{Characteristics of children hospitalised with an infectious disease (Table 1)}

The median (interquartile range (IQR)) age at first ID admission was $7.2(2.4-13.2)$ months. Compared with the ethnicity of live births in New Zealand in 2010 (27\% Māori, 11\% Pacific, 11\% Asian, and 51\% European/ other), there were higher percentages of Māori (34\%, $p<0.001)$ and Pacific $(17 \%, P<0.001)$ children among those hospitalised with an ID. By contrast, there were lower percentages of Asian (5\%, $P<0.001)$ and European/ other (43\%, $P<0.001)$ children hospitalised with an ID [19].

Of the 46,540 children hospitalised with an ID, 18,575 (40\%) lived in the most deprived households and 894 (2\%) had an underlying CCC. The most frequent season of first ID admission was winter (17,285/46,540 37\%) and the majority of first admissions were for an ARI $(25,256 / 46,54054 \%)$.

Infectious disease group and readmission risk (Table 2)

Readmission occurred at a median (IQR) of 77 (26-177) days after the first ID admission. Median (IQR) days to ID readmission was $67(25-157)$ if the first admission was ARI, 96 (23-198) if it was enteric infection, 100 (34-195) if it was an SSTI and $115(45-202)$ if it was a UTI.

Overall, 6735/10,205 (66\%) of the ID readmissions were with an ARI. In comparison with all other infections, children whose first ID admission was an ARI were at increased risk ( $\mathrm{OR}=1.87,95 \% \mathrm{CI} 1.78-1.95)$ of readmission, whereas those whose first ID admission was an enteric infection $(\mathrm{OR}=0.63,0.59-0.67)$, SSTI $(\mathrm{OR}=0.62$, 95\% CI 0.56-0.68), UTI $(\mathrm{OR}=0.83,95 \% \mathrm{CI}$ $0.74-0.94)$ or other infection $(\mathrm{OR}=0.65,95 \%$ CI $0.62-$ 0.69) were at decreased risk.

\section{Acute respiratory infection group and readmission risk (Tables 3 and 4)}

Among children whose first admission was an ARI, the risk of readmission with an ARI was increased if this first admission was with bronchiolitis ( $\mathrm{OR}=2.21$, 95\% CI 2.08-2.36) and decreased if the first admission was with pneumonia $(\mathrm{OR}=0.66,95 \%$ CI $0.61-0.73$ ), or an ARI that was not bronchiolitis, pneumonia, or influenza $(\mathrm{OR}=0.49$, 95\% CI 0.46-0.53) (Table 3). Among children whose first admission was an ARI, the principal discharge diagnosis of the readmission was lower ARI in $82 \%$. In comparison with those whose first ID admission was an upper ARI, children whose first ID admission 


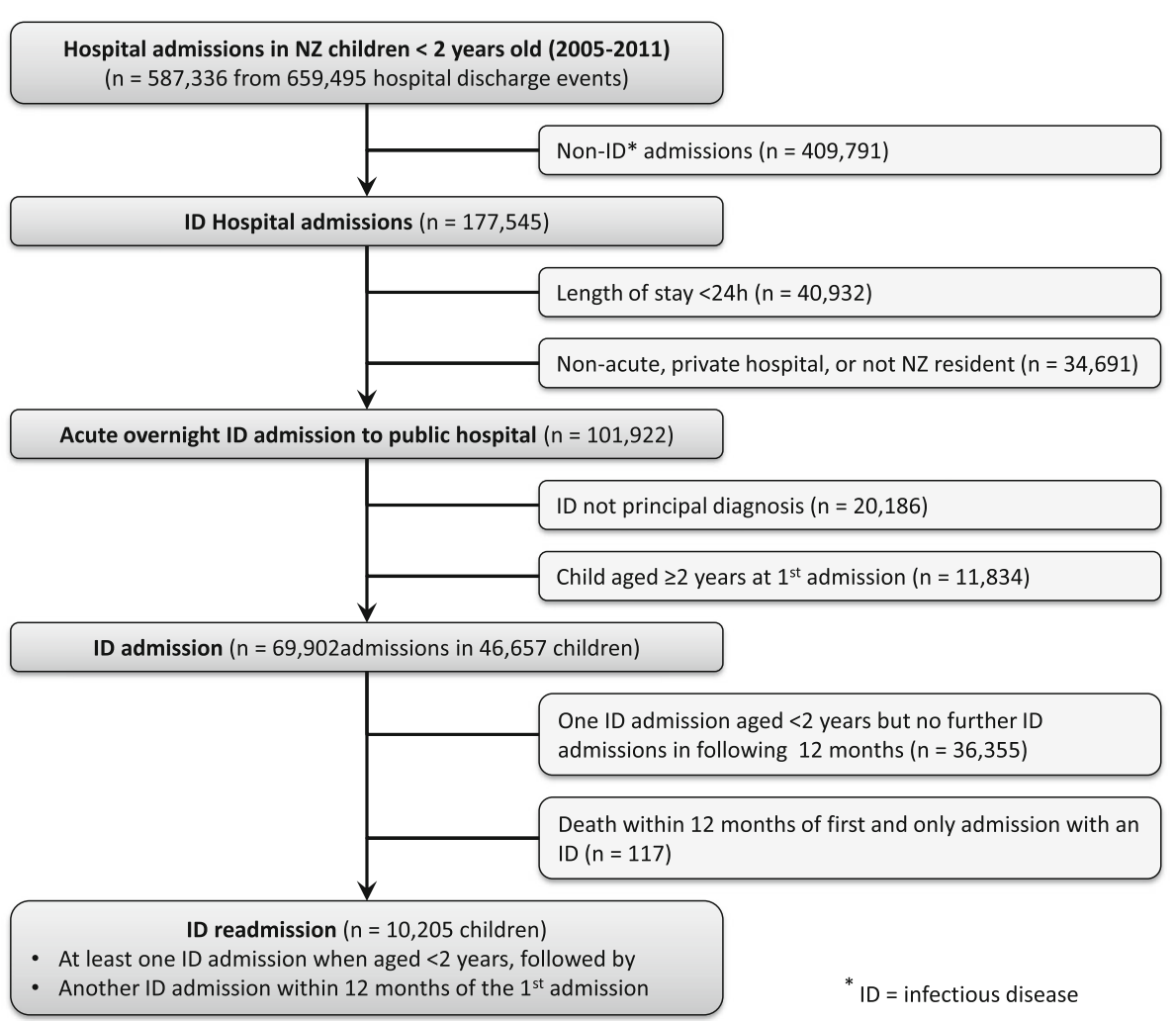

Fig. 1 Flow diagram describing study design

was a lower ARI were at increased risk of readmission with any ID (29\% vs. $19 \%, \mathrm{OR}=1.75,95 \%$ CI $1.63-1.87)$.

Among children whose first admission was with an ARI, the risk of readmission with any ID was increased if this first admission was with bronchiolitis $(\mathrm{OR}=2.27$, 95\% CI 2.17-2.38) and decreased if the first admission was with pneumonia (OR $=0.91,95 \%$ CI $0.84-0.98$ ), or an ARI that was not bronchiolitis, pneumonia, or influenza $(\mathrm{OR}=0.86,95 \%$ CI 0.81-0.92) (Table 4).

\section{Factors associated with readmission with an acute respiratory, enteric, skin or soft tissue, or urinary tract infection}

Of the 25,256 children whose first ID admission was ARI, readmission with an ARI occurred in 5521 (22\%), with median (IQR) time to readmission of 57 (24-134) days (Additional file 3). Of the 6090 children whose first ID admission was an enteric infection, readmission with an enteric infection occurred in 449 (6\%; median time to readmission 39 (5-145) days) (Additional file 4). Of the 3210 children admitted with an SSTI, readmission with an SSTI occurred in 205 (9\%; median time to readmission 54 (16-153) days) (Additional file 5). Of the 1826 children admitted with a UTI, readmission with a UTI occurred in 101 (6\%; median time to readmission 115 (45-202) days) (Additional file 6).
Children at increased risk of a second ARI admission were of younger age when first admitted with an ARI (age $<6$ months vs. 6 to 23 months old, OR $=1.62,95 \%$ CI 1.52-1.73), male gender $(\mathrm{OR}=1.20$, 95\% CI $1.13-$ 1.28), Pacific $(\mathrm{OR}=2.14,95 \% \mathrm{CI} 1.95-2.36)$ or Māori $(\mathrm{OR}=1.98,95 \%$ CI 1.82-2.14) versus European ethnicity, or living in the most $(\mathrm{OR}=1.31,95 \%$ CI 1.14-1.51) or second most $(\mathrm{OR}=1.21,95 \%$ CI 1.05-1.40) deprived compared with the least deprived quintile of households. The risk of an ARI readmission increased (OR $=1.14$, 95\% CI 1.01-1.29) for children whose first ARI hospital admission was in autumn compared with summer. Children with a CCC (OR $=3.25,95 \%$ CI 2.73-3.87) or whose first ARI admission was $\geq 3$ days in duration $(\mathrm{OR}=1.53$, 95\% CI 1.43-1.63) were at increased risk of an ARI readmission (Additional file 3).

In contrast with ARIs, demographic and illness factors were less consistently associated with the risk of readmission with a second enteric infection, SSTI or UTI (Additional files 4, 5 and 6). Children at increased risk of a second enteric infection admission were those aged < 6 months (OR $=1.53$, 95\% CI 1.24-1.87) when first admitted with an enteric infection, of male gender $(\mathrm{OR}=$ 1.25 , 95\% CI $1.03-1.52)$ or with a CCC (OR $=3.58,95 \%$ CI 2.03-6.01) (Additional file 4). Children at increased risk of a second UTI were those with a CCC $(\mathrm{OR}=3.49$, 
Table 1 Demographic and illness characteristics of children aged less than 2 years admitted to hospital with an infectious disease from 1st January 2005 to 31st December 2011

\begin{tabular}{|c|c|}
\hline & $\begin{array}{l}\text { Children ad } \\
\text { to hospital } \\
\text { an infectiou } \\
\text { before age }\end{array}$ \\
\hline Variable & $n=46,540$ \\
\hline \multicolumn{2}{|l|}{ Demographic characteristics } \\
\hline Age in months, median (IQR) & $7.2(2.4-$ \\
\hline \multicolumn{2}{|l|}{ Age, $n(\%)$} \\
\hline Less than 6-months-old & $19,028(41)$ \\
\hline 6- to 23-months-old & $27,512(59)$ \\
\hline \multicolumn{2}{|l|}{ Gender, $n(\%)$} \\
\hline Male & $26,038(56)$ \\
\hline Female & $20,502(44)$ \\
\hline \multicolumn{2}{|l|}{ Ethnicity $^{\mathrm{a}}, n(\%)$} \\
\hline Pacific & $7902(17)$ \\
\hline Māori & $15,876(34)$ \\
\hline Asian & $2499(5)$ \\
\hline Other & $740(2)$ \\
\hline European & $19,411(42)$ \\
\hline \multicolumn{2}{|l|}{ Household deprivation ${ }^{\mathrm{b}}, n(\%)$} \\
\hline Dep $9 \& 10$ (most deprived) & $18,575(40)$ \\
\hline Dep $7 \& 8$ & $10,996(24)$ \\
\hline Dep 5 \& 6 & $7226(16)$ \\
\hline Dep $3 \& 4$ & $5171(11)$ \\
\hline Dep $1 \& 2$ (least deprived) & $4435(10)$ \\
\hline \multicolumn{2}{|l|}{ Season of admissionc ${ }^{c}, n(\%)$} \\
\hline Autumn & $8586(18)$ \\
\hline Winter & $17,285(37)$ \\
\hline Spring & $13,162(28)$ \\
\hline Summer & $7507(16)$ \\
\hline \multicolumn{2}{|l|}{ Illness characteristics } \\
\hline Length of stay in days, median ( 25 th, 75 th centile) & $3(2,4)$ \\
\hline \multicolumn{2}{|l|}{ Diagnostic group of first admission, $n(\%)$} \\
\hline Acute respiratory infection (ARI) & $25,256(54)$ \\
\hline Enteric infection & $6909(15)$ \\
\hline Skin and soft tissue infection (SSTI) & $3210(7)$ \\
\hline Urinary tract infection (UTI) & $1826(4)$ \\
\hline Other & $9339(20)$ \\
\hline \multicolumn{2}{|l|}{ Presence of complex chronic conditions, $n(\%)$} \\
\hline All complex chronic conditions & $894(2)$ \\
\hline Neuromuscular & $45(0.1)$ \\
\hline Cardiovascular & $403(0.9)$ \\
\hline Respiratory & $116(0.2)$ \\
\hline Renal & $43(0.1)$ \\
\hline Gastrointestinal & $78(0.2)$ \\
\hline
\end{tabular}

Table 1 Demographic and illness characteristics of children aged less than 2 years admitted to hospital with an infectious disease from 1st January 2005 to 31st December 2011 (Continued)

\begin{tabular}{ll}
\hline & $\begin{array}{l}\text { Children admitted } \\
\text { to hospital with } \\
\text { an infectious disease } \\
\text { before age 2 years } \\
n=46,540\end{array}$ \\
Variable & $38(0.1)$ \\
\hline Haematologic and immunologic & $3(0.01)$ \\
Metabolic & $133(0.3)$ \\
Other congenital or genetic defect & $35(0.1)$ \\
Malignancy &
\end{tabular}

aEthnicity not stated, $n=112$

${ }^{\mathrm{b}}$ Area-level socio-economic deprivation was measured using the NZ Index of Deprivation (NZDep06), grouped into quintiles [18]. Data were missing for 137 (0.2\%) children

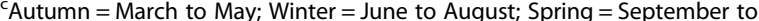
November; Summer $=$ December to February

$\mathrm{Cl}$ Confidence interval

$I Q R$ Interquartile range

95\% CI 1.45-7.51) (Additional file 6). None of these factors was associated with the risk of a second SSTI (Additional file 5).

\section{Discussion}

In this national study, $15 \%$ of children in the 2005-2009 NZ birth cohort were hospitalised with an ID in the first two years of life, $40 \%$ of whom lived in the most deprived $20 \%$ of households. Twenty-two percent of these children (3\% of the birth cohort) had at least one further ID admission within 12 months of their first admission. Two-thirds of the first ID admissions were for an ARI and over one-quarter of these children had a subsequent ARI admission. In contrast, a first ID admission for an enteric ID, SSTI or UTI was not associated with an increased risk of readmission within the same ID category.

In multivariable models, the risk factors associated with ARI readmission were younger age, male gender, Pacific or Māori ethnicity, living in a more deprived household, a first ARI admission during autumn, the presence of a CCC, or a first ARI admission of $\geq 3$ days duration. Only younger age, male gender and presence of a CCC were associated with an increased risk of enteric ID readmission, and only presence of a CCC with an increased risk of UTI readmission. None of these factors were associated with the risk of SSTI readmission.

A strength of our study was the ability to describe ID hospital admissions for the national birth cohort. In NZ, discharge data for admissions to public hospitals are loaded into the NMDS within 21 days of discharge. We are confident, therefore, that our description of ID admissions is complete. By studying a five-year birth cohort (2005-2009) we minimised the effect of an isolated 
Table 2 Risk of any infectious disease readmission within a 12 month period based upon infectious disease discharge diagnosis category at first admission

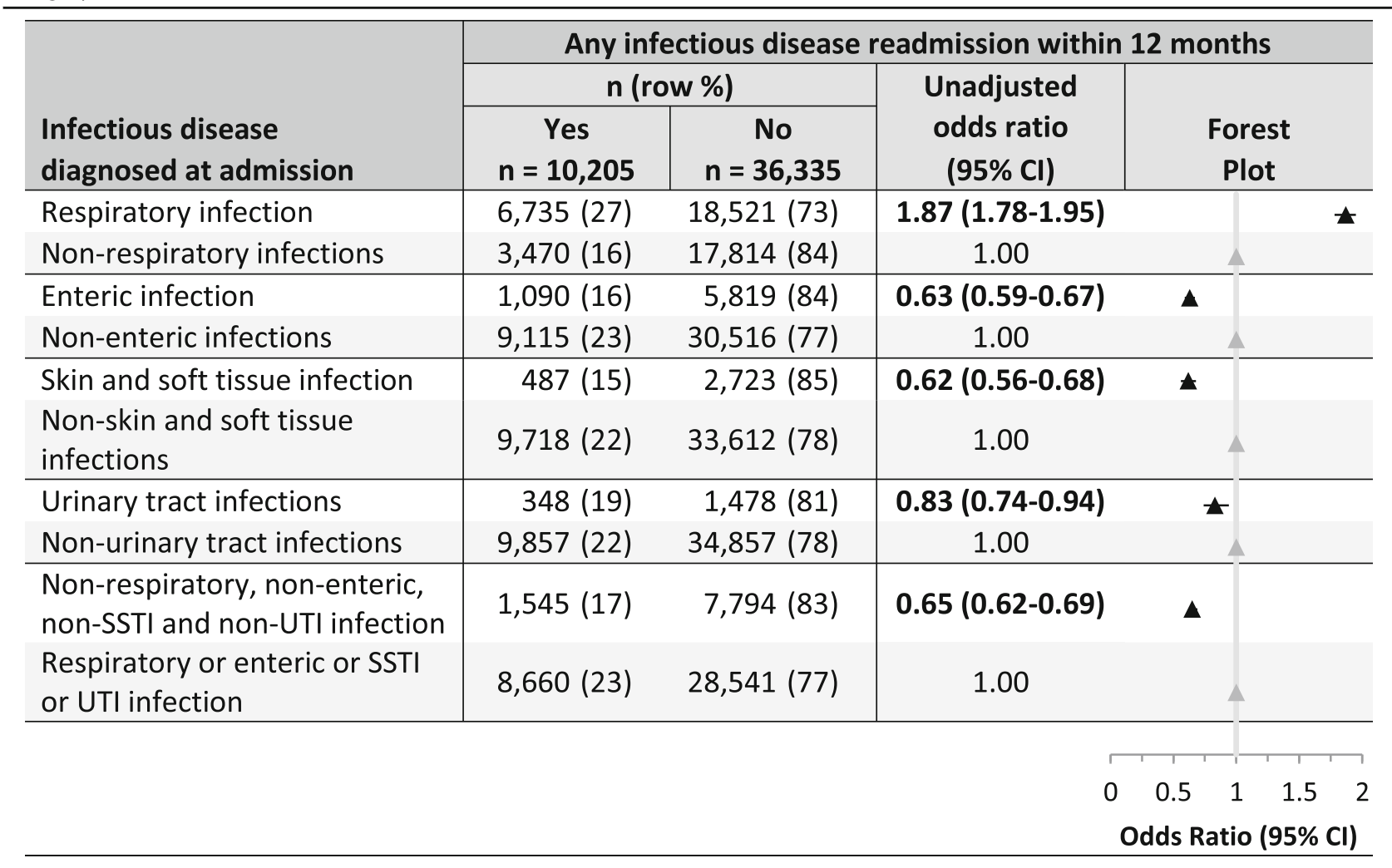

CI Confidence interval, SSTI Skin or soft tissue infection, UTI Urinary tract infection

Table 3 Risk of respiratory disease readmission within a 12 month period based upon respiratory illness present at the first admission

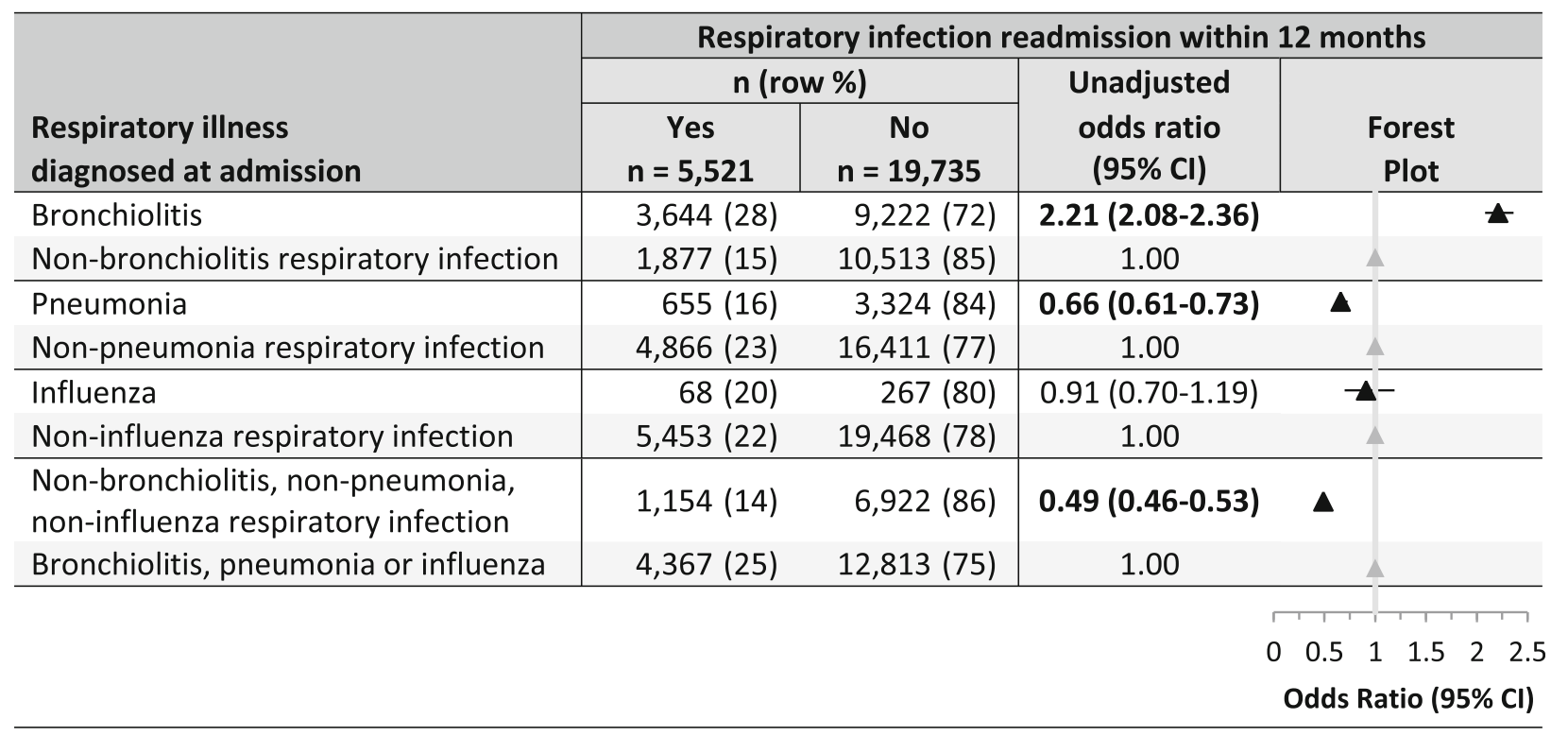


Table 4 Risk of any infectious disease readmission within a 12 month period based upon respiratory illness present at the first admission

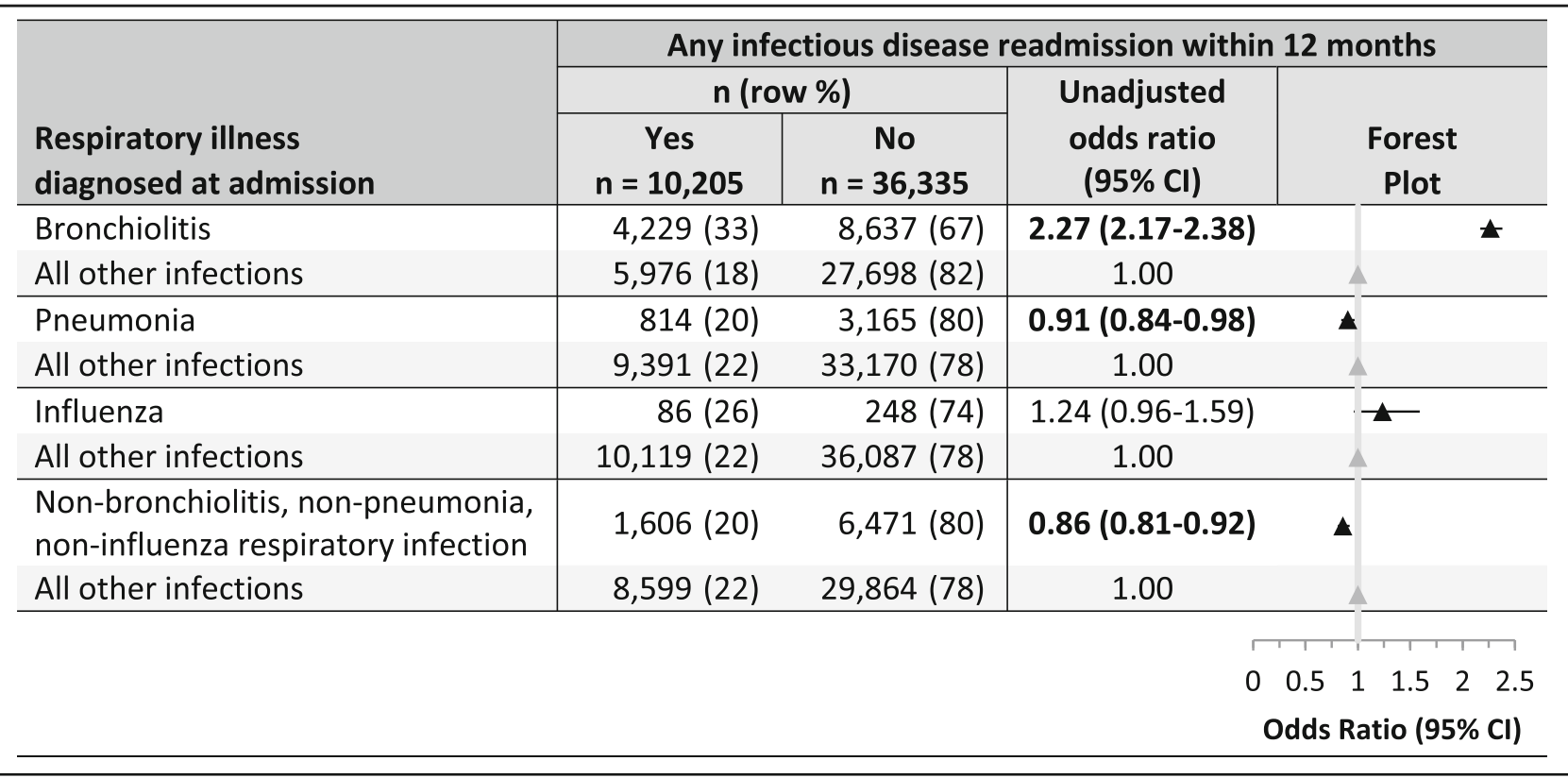

Cl Confidence interval

epidemic, for example that caused by the 2009 H1N1 influenza pandemic [20].

By limiting to ID principal discharge codes, our study underestimated the total number of ID admissions. However, limitation to principal codes was necessary to clearly describe the relationship between first and subsequent ID admissions. Our analyses were restricted to NMDS data and therefore the influence of other factors, such as cigarette smoking, or primary care received, was not evaluated, as they are not included in a national data set.

Our case definition was limited to overnight hospital admissions. This excludes very short-term hospital admissions, which may be more likely to occur due to systemic failure in the delivery of acute care in primary care and emergency departments [21].

Children with CCC and children supported with medical technology, for example a tracheostomy or ventriculo-peritoneal shunt, are especially vulnerable to events requiring hospital admission [8]. Whilst children with an underlying CCC were at an increased risk of ID readmission, in our study they accounted for only $2 \%$ of all of the children hospitalised with an ID before age 2 years.

Our data suggest that identification of children at risk of recurrent admission and intervention during the first admission may reduce total ID hospitalisation burden. Our study findings imply that such strategies should focus on preventing ARI readmission, given that the risk of ID readmission was increased for ARI but not for enteric infections, SSTIs or UTIs.
Providing specialist health care outside of the hospital is one such potential strategy. In England, the concept of "Hospital-at-Home" with paediatric nurse home care visits accessible $24 \mathrm{~h}$ per day was recently evaluated as an alternative to inpatient care for children with breathing difficulties, diarrhoea and vomiting, or fever [22]. Whilst the hospital-at-home care was well accepted by families, it did not result in any reduction in risk of hospital readmission in the subsequent 90 days [22].

Clinical pathways including specific admission and discharge criteria can help to reduce the rate of readmission within two weeks of the first admission, as shown in a recent Australian study of children $<12$ months old hospitalised with bronchiolitis [23]. This clinical pathway included improved discharge planning, including specific discharge criteria, a discharge plan developed in consultation with parents, and communication with the primary care physician [23].

Can ARI readmissions over the longer term be prevented, beyond those due to the same respiratory illness that caused the first admission? A recent meta-analysis of 25 randomised controlled trials, which enrolled 11,321 participants aged 0 to 95 years, showed that vitamin D supplementation prevents ARIs [24]. Supplementation is of most benefit to those who are vitamin D deficient, and when daily or weekly vitamin D dosing regimens without bolus doses are used [24]. In Auckland, daily vitamin $\mathrm{D}_{3}$ supplementation during pregnancy and infancy was shown to reduce the proportion of children making a primary care ARI visit up to age 18 months [25]. Therefore, vitamin $\mathrm{D}_{3}$ supplementation 
following ARI hospital admission could potentially prevent ARI readmissions. We are currently conducting a randomised clinical trial to determine if vitamin D supplementation prevents ARI healthcare visits in children under two-years-old (Trial ID: ACTRN12616000659404).

Hospital admission also provides an immunisation update opportunity. Children with more acute illness visits are at increased risk of delayed immunisations [26]. The pneumococcal conjugate vaccine prevents a proportion of ARI hospital admissions, including a spectrum of ARI without radiographic evidence of pneumonia [27]. Our study shows that infants who present in autumn are more likely to represent with ARI. Hence offering seasonal influenza vaccination prior to hospital discharge, provided the child does not have a temperature $>38{ }^{\circ} \mathrm{C}$ [28], may reduce the risk of the winter readmission of these children.

Children of Māori and Pacific ethnicity and those living in deprived households continue to be at increased risk of hospital admission and readmission with infectious diseases [2]. Characteristics of the child's home environment, such as overcrowding, dampness and mould, can increase the risk of contracting and being hospitalised with an ARI [29-32]. A recent NZ study found that the risk of ARI hospitalisation before age five years was increased for children living in households where there was gas heating in the bedroom the child slept in during their first year of life [33]. Thus, identification, during the first ARI admission, of the type of heating used in the child's household and replacing gas heating with electric heating, particularly in the room where the child sleeps, could prevent ARI readmissions.

In summary, children hospitalised with an ARI in the first 2 years of life, and in particular a lower ARI, are a group for whom strategies are required to reduce the risk of ARI readmission. Contemporary evidence indicates potential interventions including vitamin D supplementation, ensuring immunisation status is up to date and replacing gas heating in the child's bedroom. Randomised controlled trials of these interventions are necessary to determine which, if any, are sufficiently cost-effective for general implementation.

\section{Conclusions}

Hospitalisation rates for infectious diseases continue to be high for NZ children. Of the $15 \%$ of children hospitalised with an infectious disease before age two years, one-in-five will have an infectious disease readmission within 12 months of their first admission. The risk of readmission is increased when the first infectious disease is respiratory, but not when it is an enteric, skin or urinary tract infection. Strategies to prevent infectious disease readmission should focus on children hospitalised prior to age two years with lower respiratory infections.

\section{Additional files}

Additional file 1: Organ system, infectious disease diagnostic groups and associated ICD-10 codes [2, 34]. (DOCX $30 \mathrm{~kb}$ )

Additional file 2: Acute lower respiratory infection syndromes and associated ICD-10 codes [2, 34]. (DOCX $24 \mathrm{~kb}$ )

Additional file 3: Associations of demographic and illness characteristics with risk of hospital readmission with a second acute respiratory infection within 12 months of a first hospital admission with an acute respiratory infection. (DOCX $90 \mathrm{~kb}$ )

Additional file 4: Associations of demographic and illness characteristics with risk of hospital readmission with a second enteric infection within 12 months of a first hospital admission with an enteric infection. (DOCX 86 kb)

Additional file 5: Associations of demographic and illness characteristics with risk of hospital readmission with a second skin and soft tissue infection within 12 months of a first hospital admission with a skin and soft tissue infection. (DOCX $91 \mathrm{~kb}$ )

Additional file 6: Associations of demographic and illness characteristics with risk of hospital readmission with a second UTI within 12 months of a first hospital admission with a UTI. (DOCX $75 \mathrm{~kb}$ )

\section{Abbreviations}

ARI: Acute Respiratory Infection; CCC: Complex Chronic Condition; $\mathrm{Cl}$ : Confidence Interval; ICD-10: International Classification of Disease-10; ID: Infectious Disease; IQR: interquartile range; NHI: National Health Index; NMDS: National Minimum Dataset; NZ: New Zealand; OR: Odds Ratio; SSTI: Skin or Soft Tissue Infection; US: United States; UTI: Urinary Tract Infection

\section{Acknowledgements}

We acknowledge the assistance of Ilya Ratine, Senior Information Analyst, Analytical Services, NZ Ministry of Health, for assistance with accessing the National Minimum Dataset.

\section{Funding}

None.

\section{Availability of data and materials}

The Ministry of Health did not grant permission for data sharing.

\section{Authors' contributions}

SS conceived and designed the study, obtained the data, interpreted the data and prepared a first draft of the manuscript. PWR conceived and designed the study, analysed and interpreted the data, and edited the manuscript. CAG, EJB, AH, and CAC interpreted the data and edited the manuscript.CCG conceived and designed the study, communicated with the Ethics Committee, obtained the data, interpreted the data, and completed the final draft of the manuscript. All authors approved the final submitted version of the manuscript and agree to be accountable for all aspects of the work.

\section{Ethics approval and consent to participate}

The study protocol was reviewed by the NZ Health and Disabilities Ethics Committee, with the ethics committee determining that ethical approval was not required. The NZ Ministry of Health granted data access, providing data with encrypted $\mathrm{NHI}$ numbers to maintain patient anonymity.

\section{Consent for publication}

Not applicable.

Competing interests

The authors have no competing interests to declare.

\section{Publisher's Note}

Springer Nature remains neutral with regard to jurisdictional claims in published maps and institutional affiliations. 


\section{Author details}

'Paediatrics, Taranaki Base Hospital, New Plymouth, New Zealand. ${ }^{2}$ Department of Paediatrics: Child and Youth Health, Faculty of Medical and Health Sciences, University of Auckland, Private Bag 92019, Wellesley Street, Auckland 1142, New Zealand. ${ }^{3}$ Children's Research Centre, Starship Children's Hospital, Auckland, New Zealand. ${ }^{4}$ Infectious Diseases, Starship Children's Hospital, Auckland District Health Board, Auckland, New Zealand. ${ }^{5}$ Nuffield Department of Primary Care Health Sciences, University of Oxford, Oxford, England. ${ }^{6}$ Emergency Medicine, Massachusetts General Hospital, Harvard Medical School, Boston, USA. ${ }^{7}$ General Paediatrics, Starship Children's Hospital, Auckland District Health Board, Auckland, New Zealand.

Received: 18 January 2017 Accepted: 22 February 2018 Published online: 05 March 2018

\section{References}

1. Kassebaum N, Kyu HH, Zoeckler L, Olsen HE, Thomas K, Pinho C, Bhutta ZA, Dandona L, Ferrari A, Ghiwot TT, et al. Child and adolescent health from 1990 to 2015: findings from the global burden of diseases, injuries, and risk factors 2015 study. JAMA Pediatr. 2017;171(6):573-92.

2. Baker MG, Barnard LT, Kvalsvig A, Verrall A, Zhang J, Keall M, Wilson N, Wal T, Howden-Chapman P. Increasing incidence of serious infectious diseases and inequalities in New Zealand: a national epidemiological study. Lancet. 2012;379(9821):1112-9.

3. Craig E, Adams J, Oben G, Reddington A, Wicken A, Simpson J. Service NCaYE: The Health Status of Children and Young People in New Zealand. In: Department of Women's and Children's Health at the University of Otago's Dunedin School of Medicine: University of Otago; 2013.

4. Hasegawa K, Tsugawa Y, Brown DF, Mansbach JM, Camargo CA, Jr.: Trends in bronchiolitis hospitalizations in the United States, 2000-2009. Pediatrics 2013, 132(1):28-36.

5. Cheung CR, Smith H, Thurland K, Duncan H, Semple MG. Population variation in admission rates and duration of inpatient stay for bronchiolitis in England. Arch Dis Child. 2013;98(1):57-9.

6. Griffin MR, Zhu Y, Moore MR, Whitney CG, Grijalva CG. U.S. hospitalizations for pneumonia after a decade of pneumococcal vaccination. N Engl J Med. 2013;369(2):155-63.

7. Friedman B, Berdahl T, Simpson LA, McCormick MC, Owens PL, Andrews $R$, Romano PS. Annual report on health care for children and youth in the United States: focus on trends in hospital use and quality. Acad Pediatr. 2011;11:263-79.

8. Berry JG, Hall DE, Kuo DZ, Cohen E, Agrawal R, Feudtner C, Hall M, Kueser J, Kaplan W, Neff J. Hospital utilization and characteristics of patients experiencing recurrent readmissions within children's hospitals. JAMA 2011;305(7):682-90.

9. Berry JG, Toomey SL, Zaslavsky AM, Jha AK, Nakamura MM, Klein DJ, Feng JY, Shulman S, Chiang VK, Kaplan W, et al. Pediatric readmission prevalence and variability across hospitals. JAMA. 2013;309(4):372-80.

10. Brower KS, Del Vecchio MT, Aronoff SC. The etiologies of non-CF bronchiectasis in childhood: a systematic review of 989 subjects. BMC Pediatr. 2014;14(1):4.

11. Chang AB, Masel JP, Boyce NC, W G, Torzillo PJ. Non-CF Bronchiectasis: clinical and HRCT evaluation. Pediatr Pulmonol. 2003;35(6):477-83.

12. Singleton RJ, Valery PC, Morris P, Byrnes CA, Grimwood K, Redding G, Torzillo PJ, McCallum G, Chikoyak L, Mobberly C, et al. Indigenous children from three countries with non-cystic fibrosis chronic suppurative lung disease/bronchiectasis. Pediatr Pulmonol. 2014;49(2):189-200.

13. Singleton R, Morris A, Redding G, Poll J, Holck P, Martinez P, Kruse D, Bulkow LR, Petersen KM, Lewis C. Bronchiectasis in Alaska native children: causes and clinical courses. Pediatr Pulmonol. 2000;29(3):182-7.

14. Das $L$, Kovesi TA. Bronchiectasis in children from Qikiqtani (Baffin) region, Nunavut, Canada. Ann Am Thorac Soc. 2015;12(1):96-100.

15. National Health Board Business Unit. National Minimum Dataset (Hospital Events) Data Dictionary. Wellington: Ministry of Health; 2010

16. Pinner RW, Teutsch SM, Simonsen L, Klug LA, Graber JM, Clarke MJ, Berkelman RL. Trends in infectious diseases mortality in the United States. JAMA. 1996;275(3):189-93.

17. Simonsen L, Conn LA, Pinner RW, Teutsch S. Trends in infectious disease hospitalizations in the United States, 1980-1994. Arch Intern Med. 1998;158(17):1923-8.
18. Salmond C, Crampton P, Atkinson J. NZDep2006 index of deprivation Wellington: Department of Public Health, University of Otago; 2007.

19. Ministry of Health. Report on Maternity, 2010. Wellington: Ministry of Health; 2012

20. Falagas ME, Koletsi PK, Baskouta E, Rafailidis PI, Dimopoulos G, Karageorgopoulos DE. Pandemic a(H1N1) 2009 influenza: review of the southern hemisphere experience. Epidemiol Infect. 2011;139(1):27-40.

21. Gill PJ, Goldacre MJ, Mant D, Heneghan C, Thomson A, Seagroatt V, Harnden A. Increase in emergency admissions to hospital for children aged under 15 in England, 1999-2010: national database analysis. Arch Dis Child. 2013:98(5):328-34

22. Sartain SA, Maxwell MJ, Todd PJ, Jones KH, Bagust A, Haycox A, Bundred P. Randomised controlled trial comparing an acute paediatric hospital at home scheme with conventional hospital care.[see comment]. Arch Dis Child. 2002:87(5):371-5.

23. Cheney J, Barber S, Altamirano L, Medico C, Cheney M, Williams C, Jackson M, Yates P, O'Rourke P, Wainwright C. A clinical pathway for bronchiolitis is effective in reducing readmission rates. J Pediatr. 2005;147(5):622-6.

24. Martineau AR, Jolliffe DA, Hooper RL, Greenberg L, Aloia JF, Bergman P, Dubnov-Raz G, Esposito S, Ganmaa D, Ginde AA, et al. Vitamin D supplementation to prevent acute respiratory tract infections: systematic review and meta-analysis of individual participant data. BMJ. 2017:356:16583.

25. Grant CC, Kaur S, Waymouth E, Mitchell EA, Scragg R, Ekeroma A, Stewart A, Milne $T$, Rowden J, Wall CR, et al. Reduced primary care respiratory infection visits following pregnancy and infancy vitamin D supplementation: a randomised-controlled trial. Acta Paediatr. 2014;

26. Turner N, Grant C, Goodyear-Smith F, Petousis-Harris H. Seize the moments: missed opportunities to immunize at the family practice level. Fam Pract. 2009;26(4):275-8.

27. Centers for Disease Control and Prevention. Pneumonia hospitalizations among young children before and after introduction of pneumococcal conjugate vaccine-United States, 1997-2006. MMWR Morb Mortal Wkly Rep. 2009:58(1):1-4.

28. Ministry of Health: Immunisation Handbook. In: 2017; Wellington: Ministry of Health; 2017.

29. Fisk WJ, Lei-Gomez Q, Mendell MJ. Meta-analyses of the associations of respiratory health effects with dampness and mold in homes. Indoor Air. 2007;17(4):284-96.

30. Fisk WJ, Eliseeva EA, Mendell MJ. Association of residential dampness and mold with respiratory tract infections and bronchitis: a meta-analysis. Environ Health. 2010;9:72.

31. Colosia AD, Masaquel A, Hall CB, Barrett AM, Mahadevia PJ, Yogev R. Residential crowding and severe respiratory syncytial virus disease among infants and young children: a systematic literature review. BMC Infect Dis. 2012;12:95.

32. Baker MG, McDonald A, Zhang J, Howden-Chapman P. Infectious diseases attributable to household crowding in New Zealand: A systematic review and burden of disease estimate. Wellington: He Kainga Oranga/ Housing and Health Research Programme, University of Otago, 2013; 2013.

33. Tin Tin S, Woodward A, Saraf R, Berry S, Atatoa Carr P, Morton SM, Grant CC. Internal living environment and respiratory disease in children: findings from the growing up in New Zealand longitudinal child cohort study. Environ Health. 2016;15(1):120.

34. O'Sullivan CE, Baker MG. Proposed epidemiological case definition for serious skin infection in children. J Paediatr Child Health. 2010:46(4):176-83. 